\title{
ON THE EXISTENCE OF THE INVERSE OPERATION IN ALTERNATION GROUPOIDS ${ }^{1}$
}

\author{
MARLOW SHOLANDER
}

Introduction. An Abelian quasigroup $S$ may be defined as a set of elements $a, b, c, \cdots$ for which the following postulates hold:

Postulate $\mathrm{I}$. There is an equivalence relation in $S$, denoted by "=" -that is, equality of elements is reflexive, symmetric, and transitive. There is a binary operation in $S$, denoted by \# or, when convenient, by the notation of multiplication. It is understood that this implies $S$ is closed with respect to \#, and that \# is uniquely defined in $S$-that is, if $a=b$ and $c=d$, then $a c=b d$.

Postulate II. \# is an alternation-that is, if $a, b, c$, and $d$ are elements in $S,(a b)(c d)=(a c)(b d)$.

Postulate III. Each element in $S$ is proper (see $\$ 2$ for definitions).

D. C. Murdoch $[1, \text { p. 516 }]^{2}$ pointed out that an Abelian quasigroup is a natural generalization of an Abelian group. The same author [2, Theorem 11] proved that in an Abelian quasigroup it is always possible to define a new operation under which the elements of the quasigroup form an Abelian group. R. H. Bruck [3, Theorem 12] in a sense completed the theory by showing how all Abelian quasigroups may be derived from Abelian groups.

In this paper a set of elements $S$ for which postulates I and II hold is called an alternation groupoid. A series of extensions of an alternation groupoid is described which for certain groupoids leads to an Abelian quasigroup (Corollary 5.5). This imbedding process has as a special case the well known procedure for imbedding commutative semigroups in groups or, more generally, the procedure for imbedding a space $S$, in which there is a commutative and associative operation, in a space $S^{\prime}$ in such a way that each "regular" element in $S$ has an inverse in $S^{\prime}$ (see, for example, [4, p. 24]). It should be noted that A. Malcev $[5, \S 2]$ has given an example of a noncommutative semigroup which cannot be imbedded in a group.

Some interesting examples of Abelian quasigroups and alternation groupoids are given in $\$ 1$.

1. Examples of alternation groupoids. The examples of Abelian 1948.

Presented to the Society, November 30,1946; received by the editors June 12,

${ }^{1}$ A part of this paper was written while the author was receiving support from the Office of Naval Research.

${ }^{2}$ Numbers in brackets refer to references cited at the end of the paper. 
quasigroups that have appeared in print fall mainly in two classesthose based on a finite set of elements and those based on a properly chosen set of numbers by defining $x \# y$ as $a x+b y+c$, or $c x^{a} y^{b}$, where $a, b$, and $c$ are constants (in particular, \# can be ordinary addition, subtraction, multiplication, division, or the operation of taking an arithmetic or geometric mean). These examples give little indication of the variety of form found in alternation groupoids.

Moreover, the geometric interest of alternation groupoids can be given greater emphasis. For instance, each Abelian quasigroup furnishes an analogue of the theorem that the midpoints of a quadrilateral determine a parallelogram. To see this, let $S$ be the set of points in the plane and let \# be the operation of taking the midpoint. Consider the associated group operation defined by Murdoch-for fixed elements $r$ and $s$ in $S, x \circ y$ is defined as $u v$ where $x=u r$ and $y=s v$. Under 0 , the elements of $S$ form a commutative group which has $s r$ as its unit element. Thus $u r \circ s v=u v \circ s r$ and $s r, s v, u v$, and $u r$ are the vertices of a parallelogram. In the case of example $E$ below, this process gives an interesting construction for an ordinary parallelogram. From example F, we obtain an odd construction for projective addition by following the steps in the definition of operation 0 .

A. Let $S$ be the set of positive integers. Let $\alpha, \beta, \gamma$, and $\delta$ be integers such that $\alpha, \gamma, \alpha+\beta$, and $\gamma+\delta$ are positive and $\alpha \delta+\beta=\gamma \beta+\delta$. Define $x \# y$ as $\max [\alpha x+\beta, \gamma y+\delta]$.

B. Let $a$ and $b$ be points in the projective plane. Let $S$ be the set of points of the plane not on the line $a b$. Define $x \# y$ as the point of intersection of the lines $x a$ and $y b$.

C. Let $a$ be a point in the complex plane and $S$ the set of points in the plane excluding $a$ and $\infty$. Let $x \# y=\overline{x-a}(y-a) \sqrt{y-a}+a$, the reflection of $x$ with respect to the line $a y$.

D. Let $a$ and $S$ be chosen as in the preceding example but let $x \# y=(y-a) \overline{y-a} / \overline{x-a}+a$, the inverse of $x$ with respect to the circle of center $a$ which passes through $y$.

E. Let $S$ be the set of points in the Euclidean plane and $a, b$, and $c$ fixed elements of $S$. Let $x \# y$ be defined as the point $z$ which determines a triangle $x y z$ strictly similar to the triangle $a b c$ in such a way that vertices $x, y, z$ correspond respectively to vertices $a, b, c$.

F. Let $S$ be the set of points on the projective line, $s$ a fixed element of $S$, and $r$ a fixed real number. Let $x \# y$ be defined as the point $z$ for which the double ratio $(s x y z)=r$. Defined in numerical terms $x \# y=[x y-s\{x(1-r)+y r\}] /[x r+y(1-r)-s]$ and when $r=1 / 2$ we see that the arithmetic and harmonic means are special cases of this alternation. 
2. Definitions and notation. In the pages following $S$ denotes an alternation groupoid. It is well known that if $S$ has a unit element the alternation is commutative and associative and that, conversely, if an operation is commutative and associative it is an alternation.

An element $a$ in $S$ is called left regular if, for all elements $x$ and $y$ in $S, a x=a y$ implies $x=y$. In much of the material which follows we consider only "left" conditions and their consequences. We shall omit statements with corresponding "right" or "left and right" conditions. Here, for example, right regularity of an element has an obvious definition and to say an element is regular means that it is both left and right regular.

An element $a$ in $S$ is called left proper if, for all elements $b$ in $S$, $a x=b$ has a unique solution, $x$, in $S$. It follows that each element left proper in $S$ is left regular in $S$. An element $\theta$ in $S$ is called left null if $\theta x=\theta$ for all elements $x$ in $S$. If $S$ has more than one element, a left null element is not left regular.

An element, $\phi(x)$, is a power of $x$ if it belongs to the set of elements $\Phi(x)$ defined recursively by the rules:

(i). $x$ belongs to $\Phi(x)$,

(ii). If $y$ and $z$ belong to $\Phi(x), y z$ belongs to $\Phi(x)$.

Murdoch has shown that in an alternation groupoid a power of a power of $x$ is a power of $x$ and that the following laws hold:

$$
\phi(x y)=\phi(x) \phi(y), \quad \phi_{1} \phi_{2}(x)=\phi_{2} \phi_{1}(x) .
$$

An element of $S$ is said to be left regular* if all of its powers are left regular. We denote by $L, L^{*}$, and $L^{* *}$ respectively the sets of elements in $S$ which are left regular, left regular*, and left proper in $S$. The sets of elements with the corresponding right properties are denoted by $R, R^{*}$, and $R^{* *}$ respectively. Finally, we let $P=L^{* *} \cap R^{* *}$ and $Q=L^{*} \cap R^{*}$.

\section{Relations between subsets of $S$.}

THEOREM 3.1. $L^{*}$ is an alternation groupoid.

Proof. It is sufficient to show that $L^{*}$ is closed under \#. Let $\phi(a b)$ be a power of $a b$ where $a, b \in L^{*}$. Assume that for some $x, y \in S$, $\phi(a b) x=\phi(a b) y$. Then

$$
\begin{aligned}
{[\phi(a a) \phi(a a)][\phi(b b) x] } & =[\phi(a a) \phi(b b)][\phi(a a) x] \\
& =\phi\{(a a)(b b)\}[\phi(a a) x]=\phi\{(a b)(a b)\}[\phi(a a) x] \\
& =[\phi(a b) \phi(a a)][\phi(a b) x]=[\phi(a b) \phi(a a)][\phi(a b) y] .
\end{aligned}
$$

Similarly, 


$$
[\phi(a a) \phi(a a)][\phi(b b) y]=[\phi(a b) \phi(a a)][\phi(a b) y] .
$$

We may cancel to obtain $\phi(b b) x=\phi(b b) y$ and $x=y$. Hence $L^{*}$ is closed under \#.

COROLLARY 3.2. $L$ is an alternation groupoid if and only if $L=L^{*}$.

Theorem 3.3. $L^{* *}$ is an alternation groupoid.

Proof. We must show that $L^{* *}$ is closed under \#. For $h$ and $k \in L^{* *}$ and $a \in S$, we find a solution $x$ of the equation $(h k) x=a$ by choosing $y, z, w$ and $x$ so that $h y=a, h z=h, k w=y$, and $x=z w$.

Corollary 3.4. $L^{* *} \subseteq L^{*} \subseteq L$.

Proof. The second inclusion relation follows from the definitions of $L$ and $L^{*}$. Since the theorem implies that all powers of a left proper element are left proper and since a left proper element is left regular, the first inclusion relation is valid.

Theorem 3.5. If $a b \in L$, then $b \in L$. If in addition $R \neq 0, a \in L$.

Proof. If we have $b x=b y,(a b)[(a b) x]=[a(a b)](b x)=[a(a b)](b y)$ $=(a b)[(a b) y]$ and $x=y$. If $r \in R$ and $a x=a y,(a b)(x r)=(a b)(y r)$, and $x=y$.

Corollary 3.6. If $a b \in L^{*}, b \in L^{*}$. If in addition $R \neq 0, a \in L^{*}$.

Theorem 3.7. If $L^{*} \neq 0$ and $R^{*} \neq 0$, then $L^{*}=R^{*}=Q$.

Proof. Consider elements $l$ and $r$ in $L^{*}$ and $R^{*}$ respectively and $p$, a power of $r$. We first prove that $l p \in L$. Suppose $(l p) x=(l p) y$. Then

$$
\begin{aligned}
{[(l l) x][(p p)(p p)] } & =[(l p) x][(l p)(p p)] \\
& =[(l p) y][(l p)(p p)]=[(l l) y][(p p)(p p)]
\end{aligned}
$$

and $x=y$. By Theorem 3.5, $l p \in L$ implies $p \in L$. Hence $r \in L^{*}$ and $R^{*} \subseteq L^{*}$. Similarly, we prove $L^{*} \subseteq R^{*}$.

Lemma 3.8. If $h k \in L^{* *}, h(h k) \in L$.

PRoof. Let $h k=l$. If $(h l) x=(h l) y$ choose $z$ and $w$ so $l z=x, l w=y$. Then

$$
\begin{aligned}
{[(l l)(l l)][l(l z)] } & =[(l l)(l l)][(h l)(k z)] \\
& =[(l l)(h l)][(l k) x]=[(l l)(l k)][(h l) y] .
\end{aligned}
$$

Similarly, 


$$
[(l l)(l l)][l(l w)]=[(l l)(l k)][(h l) y .
$$

We cancel to obtain $z=w$. Hence $x=y$.

Theorem 3.9. If $h k \in L^{* *}, k \in L^{* *}$.

Proof. Let $l=h k$. To solve the equation $k x=a$ choose $y$ and $x$ so $l y=(h l) a$ and $l x=y$. Then $(h l)(k x)=(h k)(l x)=l y=(h l) a$. By the previous lemma, $k x=a$.

CoRollary 3.10. All elements left proper in $S$ are left proper in $L^{* *}$.

Corollary 3.11. If $L^{* *} \neq 0$ and $R^{* *} \neq 0$, then $L^{* *}=R^{* *}=P$.

Proof. To prove, say, $R^{* *} \subseteq L^{* *}$, consider $r \in R^{* *}$. Since there exists an element $x$ such that $x r=l$, it follows from the theorem that $r \in L^{* *}$.

Lemma 3.12. If $L^{* *} \neq 0, a \in L^{*}, b \in L$, and $c \in R$, then $a b \in L$ and $b c \in R$.

Proof. If $l \in L^{* *}, l$ and $a l \in L^{*}$. If $(a b) x=(a b) y$, let $l z=x$ and $l w=y$. Then $(a l)(b z)=(a l)(b w), z=w$, and $x=y$. Thus $a b \in L$. It follows as a particular case of this result that $l b \in L$. Hence if $x(b c)=y(b c)$, $(l b)(z c)=(l b)(w c), z=w$, and $x=y$.

Lemma 3.13. If $L^{* *} \neq 0$ and $R \neq 0, L \subseteq R$.

Proof. Let $b \in L$ and $c \in R$. By Lemma 3.12, $b c \in R$. By the "right" dual of Theorem 3.5, $b c \in R$ implies $b \in R$.

Lemma 3.14. If $R \neq 0, L^{* *} \subseteq R^{*}$.

Proof. Let $r \in R$ and let $\phi(l)$ be a power of $l \in L^{* *}$. By Lemma 3.12, $\phi(l) r \in R$. Hence $\phi(l) \in R$ and $l \in R^{*}$.

Theorem 3.15. If $L^{* *} \neq 0$ and $R \neq 0, L^{* *} \subseteq Q \subseteq L \subseteq R$.

PRoof. The inclusion relations are consequences of Corollary 3.4, Lemmas 3.13 and 3.14, and Theorem 3.7.

Theorem 3.16. If $P \neq 0, P \subseteq Q=L=R$.

Proof. If $P \neq 0$, neither $L$ nor $R^{* *}$ is null. By the dual of Theorem 3.15, $R^{* *} \subseteq Q \subseteq R \subseteq L$. From these relations, from Theorem 3.15, and from Corollary 3.11, the conclusion of this theorem follows.

As an immediate consequence of our definitions, we have the following relations.

THEOREM 3.17. If $S_{1}$ and $S_{2}$ are alternation groupoids and $S_{1} \subseteq S_{2}$, then $L_{1} \supseteq S_{1} \cap L_{2}$ and $L_{1}^{*} \supseteq S_{1} \cap L_{2}^{*}$. 
4. The imbedding theorem. This section is devoted to a proof of Theorem 4.1. If $S$ has no left regular* element we may satisfy the theorem by choosing $S_{\infty}$ as $S$. Hence throughout the section we assume $L^{*} \neq 0$. Notations such as $L_{\infty}^{*}$, the set of left regular* elements in $S_{\infty}$, have an obviously meaning. We show in Theorem 4.11 that the following theorem cannot be made stronger by replacing left regularity* with mere left regularity.

TheOREM 4.1. An alternation groupoid $S$ can be imbedded in an alternation groupoid $S_{\infty}$ with the following properties:

(A). $L^{*}$ is isomorphic to a subset of $L_{\infty}^{*}$.

(B). Left regular* elements in $S_{\infty}$ are left proper in $S_{\infty}$.

(C). $S_{\infty}$ is a minimal extension of $S$ into an alternation groupoid with properties $\mathrm{A}$ and $\mathrm{B}$.

Definition. $S_{1}$, the first left extension of $S$, is a groupoid whose elements are ordered pairs $(a, b)$, where $a \in L^{*}$ and $b \in S$. In $S_{1}$ we define a binary operation \# and the equality of elements as follows:

(i). $(a, b) \#(c, d)=(a c, b d)$.

(ii). $(a, b)=(c, d)$ if and only if, in $S,(a a) d=(a c) b$.

When the notation $(a, b)$ is used it is assumed $a$ is left regular*. By $\phi(a, b)$ we shall mean $\phi((a, b))$. This power obviously has the value $(\phi(a), \phi(b))$.

\section{LEMMA 4.2. \# is a binary operation in $S_{1}$.}

Proof. $S_{1}$ is clearly closed with respect to \#. To show the operation is uniquely defined, we assume $(a, b)=(c, d)$ and $(e, f)=(g, h)$. It follows that

$$
[(a e)(a e)](d h)=[(a a) d][(e e) h]=[(a c) b][(e g) f]=[(a e)(c g)](b f),
$$

$(a e, b f)=(c g, d h)$, and $(a, b)(e, f)=(c, d)(g, h)$.

Lemma 4.3. If $(a, b)=(c, d)$, then $(x a) d=(x c) b$ for all $x$ in $S$. Conversely, if $x \in L^{*}$ and $(x a) d=(x c) b$, then $(a, b)=(c, d)$.

Proof. Since $(a a) d=(a c) b$,

$$
[(a a) a][(x c) b]=[(a x) a][(a c) b]=[(a x) a][(a a) d]=[(a a) a][(x a) d] \text {. }
$$

Since $(a a) a$ is left regular, the first statement is proved. The second statement has a similar proof.

LEMMA 4.4. The relation " $="$ is an equivalence relation in $S_{1}$.

Proof. That the equality is reflexive is trivial; that it is symmetric 
follows from the previous lemma. To prove transitivity, assume $(a, b)=(c, d)$ and $(c, d)=(e, f)$. It follows that

$$
\begin{aligned}
{[(c c)(c c)][(a a) f] } & =[(c c)(a a)][(c c) f] \\
& =[(c a)(c a)][(c e) d]=[(c a)(c e)][(c a) d] \\
& =[(c c)(a e)][(c c) b]=[(c c)(c c)][(a e) b] .
\end{aligned}
$$

Cancellation of $(c c)(c c)$ gives the condition that $(a, b)=(e, f)$.

We have shown that $S_{1}$ satisfies postulate I. We can show without difficulty that postulate II is also satisfied, and we state the following theorem by way of summary.

\section{Theorem 4.5. $S_{1}$ is an alternation groupoid.}

Theorem 4.6. $(a, x) \in L_{1}^{*}\left[\right.$ resp. $\left.R_{1}^{*}\right]$ if and only if $x \in L^{*}\left[\right.$ resp. $\left.R^{*}\right]$.

Proof. Proofs of both statements are similar-that the conditions are sufficient is easily seen. To prove necessity assume, say, $x \in L^{*}$. There exist elements $y$ and $z$ and a power of $x$ such that $y \neq z$ but $\phi(x) y=\phi(x) z$. Then $\phi(a, x)(a, y)=(\phi(a) a, \phi(x) y)=(\phi(a) a, \phi(x) z)$ $=\phi(a, x)(a, z)$. If $\phi(a, x) \in L_{1},(a, y)=(a, z)$ and $y=z$. Hence $\phi(a, x)$ $\notin L_{1}$ and $(a, x) \notin L_{1}^{*}$.

Corollary 4.7. $R_{1}^{*}=0$ if and only if $R^{*}=0$.

Lemma 4.8. $(a, a x)=(b, b y)$ if and only if $x=y$.

THEOREM 4.9. If $e \in L^{*}$, the correspondence $x \sim(e, e x)$ defines an isomorphism of $S$ to a subset of $S_{1}$ under which $L^{*}$ is isomorphic to a subset of $L_{1}^{*}$.

PROOF. That the correspondence determines an isomorphism follows from Lemma 4.8. From Theorems 3.1 and 4.6 and from Corollary 3.6, $(e, e x) \in L_{1}^{*}$ if and only if $x \in L^{*}$.

Whenever it proves convenient, we identify $S$ with the subset of $S_{1}$ to which it is isomorphic.

We have shown that $S_{1}$ satisfies property (A) of Theorem 4.1. That we cannot expect it to satisfy properties (B) and (C) follows from consideration of the example where $S$ is the set of real numbers on a finite interval and where $x \# y$ is defined as $(x+y) / 2 . S_{1}$ does, however, satisfy a weakened form of Theorem 4.1 given below.

THEOREM 4.10. $S_{1}$ can be uniquely characterized as a minimal extension of $S$ into an alternation groupoid which satisfies property (A) of Theorem 4.1 and the following property: If $a \in L^{*}$ and $b \in S$, then $a x=b$ has a solution $x$ in $S_{1}$. 
Proof. Since $(e, e a)(a, b)=(e, e b),(a, b)$ is the required solution. If $S$ is imbedded in a space $T$ with this property and property (A), let $T_{1}$ be the subset of $T$ made up of elements which are solutions of the equations $a x=b$ where $a \in L^{*}$ and $b \in S$. Each element of $T_{1}$ can be represented as an ordered pair $[a, b]$. It is a routine matter to show that the correspondence $(a, b) \sim[a, b]$ maps $S_{1}$ isomorphically on $T_{1}$.

Definition. $S_{n}$, the $n$th left extension of $S$, is defined as the first left extension of $S_{n-1}, n=2,3, \cdots . S_{\infty}$, the left extension of $S$, is defined as the union of the spaces $S_{n}$.

Thus by repeating the imbedding process just discussed, we obtain $S \subseteq S_{1} \subseteq S_{2} \subseteq \cdots \subseteq S_{\infty}$. It is evident that $S_{\infty}$ is an alternation groupoid and that $L^{*} \subseteq L_{\infty}^{*}$. If $\alpha \in L_{\infty}^{*}$ and $\beta \in S_{\infty}$, there exists (see Theorem 4.9) an integer $n$ such that $\alpha \in L_{n}^{*}$ and $\beta \in S_{n}$. By Theorem 4.10, $\alpha x=\beta$ has a solution $x$ in $S_{n+1} \subseteq S_{\infty}$ and thus $\alpha \in L_{\infty}^{* *}$. Finally it is not difficult to show $S_{\infty}$ is the minimal extension described in Theorem 4.1 , and the proof of that theorem is now complete.

If we measure the worth of an extension $T$ of $S$ by the number of elements of $S$ which are left proper in $T$, the following theorem shows that the left extension of $S$, described in this section, is the best possible. The theorem is a direct consequence of Theorem 3.17 and Corollary 3.4.

Theorem 4.11. There exists no extension of an alternation groupoid $S$ into an alternation groupoid $T$ such that an element not left regular* in $S$ is left proper, or even left regular*, in $T$.

THEOREM 4.12. The properties of being right regular*, right proper and left proper are preserved under left extension.

Proof. That right regularity* is preserved follows from Theorems 3.7 and 4.6 (it can be shown that if $L^{* *} \neq 0$, even right regularity is preserved). To show right properness is preserved it will be sufficient to find an element of $S_{1}$ which when multiplied on the right by $(e, e r)$ gives $(a, f)$, where $r \in R^{* *}, e$ and $a \in L^{*}$, and $f \in S$. Choose $g$ and $d$ so $f=g(r r)$ and $a=d r$. Applying Theorem 3.7 and the dual of Corollary 3.6, we have $a \in R^{*}, d \in R^{*}$, and $d \in L^{*}$. Hence $(d, g)$ is an element in $S_{1}$. It proves to be the element we sought. That left properness is preserved is proved similarly.

Before leaving this section we note the inclusion relations which have been established by Theorems 3.17, 4.1, 4.12, and Corollary 3.4.

THEOREM 4.13. $L^{* *} \subseteq L_{\infty}^{* *}=L_{\infty}^{*}, R^{* *} \subseteq R_{\infty}^{* *}, L^{*}=S \cap L_{\infty}^{*}$, and $R^{*}=S$ $\cap R_{\infty}^{*}$. 
5. General extensions of $S$. It is necessary to introduce new notation. Let $S_{0}$ be $S$. Let $S_{\infty 0}$ be $S_{\infty}$ and let $S_{n 0}$ be $S_{n}$, the $n$th left extension of $S$. Let $S_{0 \infty}$ and $S_{0 n}$ be respectively the right extension and the $n$th right extension of $S$. Finally let $S_{m n}$ be $\left(S_{m 0}\right)_{0 n}$ where $m$ and $n$ are any non-negative integers or where either $m$ and $n$ or both are replaced by the symbol $\infty$. It is evident that $S_{n+10}=\left(S_{n 0}\right)_{10}$ and $S_{0 n+1}=\left(S_{0 n}\right)_{01}$. It is consistent with what we have done in $\$ 4$ to define $S_{10}=S\left[S_{01}=S\right]$ in case $L^{*}=0\left[R^{*}=0\right]$.

LemMa 5.1. $S_{11}$ is isomorphic to $\left(S_{01}\right)_{10}$.

Proof. If $L^{*}=0, L_{01}^{*}=0$ by the dual of Corollary 4.7 , and $\left(S_{01}\right)_{10}$ $=S_{01}=\left(S_{10}\right)_{01}=S_{11}$. Similarly if $R^{*}=0, S_{11}=\left(S_{01}\right)_{10}$. We now assume $L^{*}=R^{*} \neq 0$ (see Theorem 3.7) and let $x_{n} \in S_{11}$. We define a correspondence $x_{n} \sim y_{n}$ where $y_{n} \in\left(S_{01}\right)_{10}$ by means of six equations. By Theorem 4.10 and its dual, there exist elements $r_{n}$ in $R_{10}^{*}, p_{n}$ in $S_{10}, a_{n}$ and $b_{n}$ in $L^{*}$, and $c_{n}$ and $g_{n}$ in $S$, such that $x_{n} r_{n}=p_{n}$ in $S_{11}, a_{n} r_{n}=c_{n}$ in $S_{10}$, and $b_{n} p_{n}=g_{n}$ in $S_{10}$. Since $a_{n}$ and $r_{n}$ are regular* in $S_{10}, c_{n}$ is regular* in $S_{10}$ and hence in $S$ (see Theorems 3.1, 3.17, and 4.12). Let $s_{n}$ and $q_{n}$ be elements in $S_{01}$ such that $s_{n} a_{n}=b_{n}$ and $q_{n} c_{n}=g_{n}$ in $S_{01}$. By the dual of Corollary 3.6, $s_{n}$ is right regular* and hence regular* in $S_{01}$. There exists an element $y_{n}$ in $\left(S_{01}\right)_{10}$ such that $s_{n} y_{n}=q_{n}$ in $\left(S_{01}\right)_{10}$. That a different choice of elements $r_{n}, p_{n}, a_{n}, b_{n}, c_{n}$ and $g_{n}$ will lead to the same element $y_{n}$ will follow from our proof that $x_{m}=x_{n}$ if and only if $y_{m}=y_{n}$. We apply repeatedly Lemma 4.3 , Theorem 4.10 , and their duals. Assume, say, $x_{1}=x_{2}$. By the definition of equality (in the 1st right extension of $\left.S_{10}\right), p_{2}\left(r_{1} r_{1}\right)=p_{1}\left(r_{2} r_{1}\right)$ in $S_{10}$. But

$$
\left[b_{1}\left(a_{2} a_{1}\right)\right]\left[p_{1}\left(r_{2} r_{1}\right)\right]=g_{1}\left(c_{2} c_{1}\right)
$$

and

$$
\left[b_{2}\left(a_{1} a_{1}\right)\right]\left[p_{2}\left(r_{1} r_{1}\right)\right]=g_{2}\left(c_{1} c_{1}\right) .
$$

Hence, in $S$,

$$
\left[\left\{b_{1}\left(a_{1} a_{1}\right)\right\}\left\{b_{1}\left(a_{2} a_{1}\right)\right\}\right]\left\{g_{2}\left(c_{1} c_{1}\right)\right\}=\left[\left\{b_{1}\left(a_{1} a_{1}\right)\right\}\left\{b_{2}\left(a_{1} a_{1}\right)\right\}\right]\left\{g_{1}\left(c_{2} c_{1}\right)\right\}
$$

or, restated,

$$
\left\{\left(b_{1} b_{1}\right) g_{2}\right\}\left[\left\{\left(a_{1} a_{2}\right) c_{1}\right\}\left\{\left(a_{1} a_{1}\right) c_{1}\right\}\right]=\left\{\left(b_{1} b_{2}\right) g_{1}\right\}\left[\left\{\left(a_{1} a_{1}\right) c_{2}\right\}\left\{\left(a_{1} a_{1}\right) c_{1}\right\}\right] .
$$

This equality implies that the solutions $\alpha$ and $\beta$ of the equations

$$
\begin{aligned}
\alpha\left[\left(a_{1} a_{1}\right) c_{2}\right] & =\left(b_{1} b_{1}\right) g_{2}, \\
\beta\left[\left(a_{1} a_{2}\right) c_{1}\right] & =\left(b_{1} b_{2}\right) g_{1}
\end{aligned}
$$


are equal in $S_{01}$. These solutions are $\left(s_{1} s_{1}\right) q_{2}$ and $\left(s_{1} s_{2}\right) q_{1}$. Their equality implies that $y_{1}=y_{2}$ in $\left(S_{01}\right)_{10}$. Since these steps are reversible, the correspondence of $x_{n}$ to $y_{n}$ is one to one. It remains to prove that the correspondence is an isomorphism. If $x_{1} \sim y_{1}$ and $x_{2} \sim y_{2}$, let $x_{1} x_{2}=x_{3}$, $r_{1} r_{2}=r_{3}, p_{1} p_{2}=p_{3}$, and so on. It follows that $x_{1} x_{2}=x_{3} \sim y_{3}=y_{1} y_{2}$.

For convenience, we identify $S_{11}$ with $\left(S_{01}\right)_{10}$ and use equality below instead of an isomorphism notation.

LemMA 5.2. $\left(S_{0 n}\right)_{m 0}=\left(S_{m 0}\right)_{0 n}$ for $m, n=0,1,2, \cdots$.

Proof. As in Lemma 5.1 we may assume $L^{*}=R^{*} \neq 0$. Clearly the equality holds if either $m$ or $n=0$. We assume $m \neq 0$ and $n \neq 0$ and proceed by induction from the result of Lemma 5.1. If $S_{h 1}=\left(S_{01}\right)_{h 0}$, then $S_{h+11}=\left(S_{h+10}\right)_{01}=\left[\left(S_{h 0}\right)_{10}\right]_{01}=\left[\left(S_{h 0}\right)_{01}\right]_{10}=\left[\left(S_{01}\right)_{h 0}\right]_{10}=\left(S_{01}\right)_{h+10} 0$, and $S_{m 1}=\left(S_{01}\right)_{m 0}$ for $m=1,2, \cdots$ Similarly, if $S_{m h}=\left(S_{0 h}\right)_{m 0}$, $S_{m h+1}=\left(S_{0 h+1}\right)_{m 0}$, and the lemma is proved.

THEOREM 5.3. The right extension of the left extension of $S$ is isomorphic to the left extension of the right extension of $S$.

Proof. It is easy to validate the two equalities below which have not yet been established. $S_{\infty \infty}=\left(S_{\infty 0}\right)_{0 \infty}=\bigcup_{n}\left(U_{m} S_{m 0}\right)_{0 n}=\bigcup_{n} \bigcup_{m}\left(S_{m 0}\right)_{0 n}$ $=U_{m} U_{n}\left(S_{0 n}\right)_{m 0}=\bigcup_{m}\left(U_{m} S_{0 n}\right)_{m 0}=\left(S_{0 \infty}\right)_{\infty}$.

The following theorem follows principally from Theorems 4.1, 4.13 , and their duals.

THEOREM 5.4. In Theorem 4.1 we may replace $S_{\infty}$ by $S_{\infty \infty}$ and left regularity* properties by the corresponding regularity* properties.

Corollary 5.5. An alternation groupoid $S$ may be imbedded in an Abelian quasigroup if and only if all elements of $S$ are regular.

We may classify alternation groupoids according to whether or not they have left regular* or right regular* elements. Members in different classes, as we have seen, have distinctly different types of extensions.

I. $L^{*}=R^{*}=0$.

Here $S_{\infty \infty}=S$ has no left or right proper element. An example: example A of $\S 2$ with $\alpha=5, \beta=-2, \gamma=3$, and $\delta=-1$. In this example, 1 is regular and $2 \in R$.

II. $L^{*} \neq 0, R^{*}=0$.

Here $S_{\infty \infty}=S_{\infty}$ and in $S_{\infty}$ we have $L_{\infty}^{* *}=L_{\infty}^{*} \subseteq L_{\infty}$ and $R_{\infty}=0$. Example: let $S$ be the set of points $(x, y)$ in the square $-1 \leqq x \leqq 1$, $-1 \leqq y \leqq 1$ and define $\left(x_{1}, y_{1}\right) \#\left(x_{2}, y_{2}\right)$ as $\left(x_{1} / 2+x_{2} / 2, y_{2}\right)$.

III. The situation dual to that of II. 
IV. $L^{*}=R^{*} \neq 0$.

Here $S_{\infty \infty \infty}$ is such that $P_{\infty \infty \infty}=Q_{\infty \infty \infty}=L_{\infty \infty \infty}=R_{\infty \infty \infty}$. (If $S_{\infty} \neq S_{\infty \infty \infty}, L_{\infty}^{* *}$ $=Q_{\infty} \subseteq L_{\infty} \subseteq R_{\infty}$ and $R_{\infty}^{* *}=0$.) Example: example A of $\S 2$ with $\alpha=3$, $\beta=-2, \gamma=2$, and $\delta=-1$. In this example, $1 \in Q$ and $2 \in R$.

6. Conditions which insure that $L=L^{*}$. Since a necessary and sufficient condition that $S$ have an extension with left proper elements is that $L^{*} \neq 0$, it is of interest to find what conditions insure that left regular elements are left regular* or, in other words, that the left regular elements are closed under \#. This closure property holds in many special alternation groupoids-for example, an idempotent groupoid or a groupoid in which \# is associative. The following theorem shows in general how badly an alternation groupoid must behave if $L \neq L^{*}$.

THEOREM 6.1. If $S$ is an alternation groupoid in which $L \neq 0$ and $L \neq L^{*}$, then:

(1). $S$ is infinite.

(2). There is no proper element in $S$.

(3). If $S$ has a right proper element, $S$ has left regular elements which are not right regular.

(4). If $S$ has a left proper element, then either $S$ has no right regular element or $S$ has right regular elements which are not left regular.

(5). S has left regular elements whose squares are not left regular.

(6). There are elements in $S$ neither left regular nor left null.

Proof. One proves property (1) just as one proves every finite integral domain is a field. Assume $S$ contains a finite number of elements $x_{1}, x_{2}, x_{3}, \cdots, x_{n}$ where $x_{1}$, say, $\in L$. Since no two elements in the set $x_{1} x_{1}, x_{1} x_{2}, \cdots, x_{1} x_{n}$ are equal, this set consists of the elements of $S$ in some order. Hence $x_{1} \in L^{* *}$, and by Corollary 3.4, $x_{1} \in L^{*}$.

Property (2) is a direct consequence of Theorem 3.16. To prove property (5) we show that if all squares of left regular elements are left regular, $L$ is closed under \#. Let $a$ and $b \in L$ and let $(a b) x=(a b) y$. Then $[(a a)(a a)][(b b) x]=[(a b)(a a)][(a b) x]=[(a a)(a a)][(b b) y]$. Since $b b, a a$, and $(a a)(a a) \in L, x=y$.

We prove property (3) by showing that if it does not hold property (5) does not hold. Let $a \in L$ and assume $L \subseteq R$. Then, by the dual of Lemma 3.12, $a a \in L$. Property (4) is proved similarly. Property (6) is a consequence of Theorem 6.6 to a proof of which the remainder of this section is devoted.

Let $N, N_{1}$ and $N_{2}$ denote respectively the sets of null, left null, and right null elements in $S$. The following lemma holds for any groupoid satisfying postulate I of the introduction. 
LemmA 6.2. If $N_{1} \neq 0$ and $N_{2} \neq 0$, there exists a null element $\theta$ and $N=N_{1}=N_{2}=(\theta)$.

Proof. If $\theta_{1} \in N_{1}$ and $\theta_{2} \in N_{2}, \theta_{1}=\theta_{1} \theta_{2}=\theta_{2}$. Thus each left null element equals every right null element and, in particular, all null elements are equal.

LEMMA 6.3. If an alternation groupoid $S$ has more than one element and if $a b \in L$, then neither $a$ nor $b \in N_{1}$.

Proof. If $a \in N_{1}, a b=a \in N_{1}$. If $b \in N_{1},(a b)[(a b) a]=[a(a b)](b a)$ $=[a(a b)](b b)=(a b)[(a b) b]$ and $a=b \in N_{1}$.

LEMMA 6.4. If $S=L \cup N_{1}$, if $N_{1} \neq 0$, and if $N_{1} \neq S$, there exists a null element $\theta$ in $S$.

Proof. Let $x \in S, a \in L, \theta_{1}$ and $\theta_{2} \in N_{1}$. By Lemma 6.3, $x \theta_{1}$ and $x \theta_{2}$ $\in N_{1}$. Hence $a \theta_{1}=\left(a \theta_{1}\right)\left(\theta_{2} a\right)=\left(a \theta_{2}\right)\left(\theta_{1} a\right)=a \theta_{2}$, and $\theta_{1}=\theta_{2}$. Thus all left null elements are equal, say, to $\theta$. In particular $x \theta$, being left null, equals $\theta$.

As a corollary to Lemmas 6.2 and 6.4 we have:

LEMma 6.5. If $S=L \cup N_{1}$, if $L \neq 0$, and if $L \neq S$, there exists a null element $\theta$ such that $L \cup(\theta)=S$.

Theorem 6.6. If $S=L \cup N_{1}, L=L^{*}$.

Proof. If $L=0$ or $L=S$, the theorem holds. If neither equality holds, $N_{1}=(\theta)$ by Lemma 6.5. Then $a$ and $b \in L$ implies $a b \in L$, for if $a b=\theta, a b=a \theta$, and $b=\theta$.

\section{REFERENCES}

1. D. C. Murdoch, Quasi-groups which satisfy certain generalized associative laws, Amer. J. Math. vol. 61 (1939) pp. 509-522.

2. - Structure of abelian quasigroups, Trans. Amer. Math. Soc. vol. 49 (1941) pp. 392-409.

3. R. H. Bruck, Some results in the theory of quasigroups, Trans. Amer. Math. Soc. vol. 55 (1944) pp. 19-52. 1942.

4. N. Bourbaki, Algèbre, Actualités Scientifiques et Industrielles, no. 934, Paris,

5. A. Malcev, On the immersion of an algebraic ring into a field, Math. Ann. vol. 113 (1936) pp. 686-691.

BROWN UNIVERSITY AND

WASHINGTON UNIVERSITY 